\title{
Acute Porphyria Presenting with Hyperamylasemia
}

\author{
P. MARK GLENCROSS, MD, DAVID D. LEDINGHAM, MD, FACS, \\ and JEFFREY A. ALEXANDER, MD
}

KEY WORDS: hyperamylasemia; hyperlipasemia; acute porphyria.

An elevation of serum amylase and lipase has not been reported previously to occur with porphyria. The current report describes a patient who presented with the clinical and laboratory picture of acute pancreatitis: elevated amylase, lipase, amylase-creatinine clearance ratio, and with abdominal pain. Only after extensive evaluation was the patient found to have acute porphyria without evidence of coexistent pancreatitis. Hyperamylasemia on two separate hospitalizations was seen to resolve with treatment of her porphyria.

\section{CASE REPORT}

A 23-year-old white female with a history of Crohn's disease who had had no abdominal pain in five years presented with colicky right lower quadrant and right pelvic pain that developed over two months' time. After colonoscopy, small bowel x-ray, and CT scan of the abdomen and pelvis failed to reveal the cause of her pain, she underwent laparotomy. Abdominal adhesions were lysed, and a right ovarian endometrioma was removed. Postoperatively this colicky lower abdominal pain was resolved, and she made an uneventful postoperative recovery and was discharged on the eighth postoperative day. She returned 20 days postoperatively with diffuse, constant abdominal pain that developed over $12 \mathrm{hr}$. Her last bowel movement was $36 \mathrm{hr}$ prior to admission; this was formed and without blood. She had a history of a partial ileal resection for obstruction secondary to Crohn's disease (pathology slides reviewed were consistent with Crohn's disease) six years ago. Five years ago, the patent underwent an abdominal exploration for a three-day history of acute abdominal pain that found no evident intraabdominal pathology. She had no history of

Manuscript received June 19, 1990; revised manuscript received October 11, 1990; accepted October 15, 1990.

From the Departments of Medicine and Surgery, Burns Clinic Medical Center, and Department of Medicine, University of Michigan Medical Center.

Address for reprint requests: Dr. Jeffrey A. Alexander, Burns Clinic Medical Center, 560 West Mitchell, Petoskey, Michigan 49770 . alcohol abuse, recent alcohol use, or previous pancreatitis. Her only oral medication was oral contraceptives.

Physical examination revealed a regular pulse of 130 , a temperature of $98.9^{\circ} \mathrm{F}$, and a blood pressure of $150 / 90$. The patient was a thin white female in moderate distress. Her abdominal exam showed diffuse tenderness to palpation without rebound tenderness and with normal bowel sounds. Abdominal roentgenograms were consistent with an ileus. Serum amylase and lipase were 520 and 63 , respectively, and remained elevated for the next four weeks (Table 1). An amylase-creatinine clearance ratio was $5.4 \%$ (normal: 1-4\%). Multiple serum calcium measurements corrected for hypoalbuminemia, ionized serum calcium, serum parathyroid hormone, and multiple serum triglyceride measurements were within the normal range. The patient received only diazepam, chlorpromazine, and central hyperalimentation. Barium small bowel and colon exams revealed only the surgical small bowel colonic anastomosis and hypomotility. Ultrasound examination showed no common bile duct dilatation and no common bile duct or gallbladder stones. Three CT scans suggested "edema" of the pancreas; however, no pancreatic phlegmon, pseudocyst, or extrapancreatic fluid collections were seen. An ERCP examination of the common bile duct and pancreatic duct was normal.

Over the four weeks after admission, the patient continued to have abdominal pain and persistent elevation of her serum amylase and lipase. After developing spiking fevers, she underwent an abdominal exploration, which revealed completely normal viscera, including pancreas. She was unable to be weaned from the ventilator postoperatively. She also developed severe gastric atony, progressive neuropathy, and bullous skin lesions. An EMG/ NCV study documented an extensive primarily motor axonal neuropathy. Biopsies of the skin lesions revealed a noninflammatory, subepidermal blister without immunoglobulin staining, consistent with porphyria. Stool and urine quantitative porphyrins were found to be elevated (Table 1), consistent with porphyria. Hematin pulse therapy, oral $\beta$-carotene, and high carbohydrate intravenous feedings were initiated after transfer to the University of Michigan Medical Center. With therapy the patient was eventually extubated and had improvement in her skin lesions and abdominal pain along with decreases in her serum amylase and lipase. Urinary porphyrins had a 


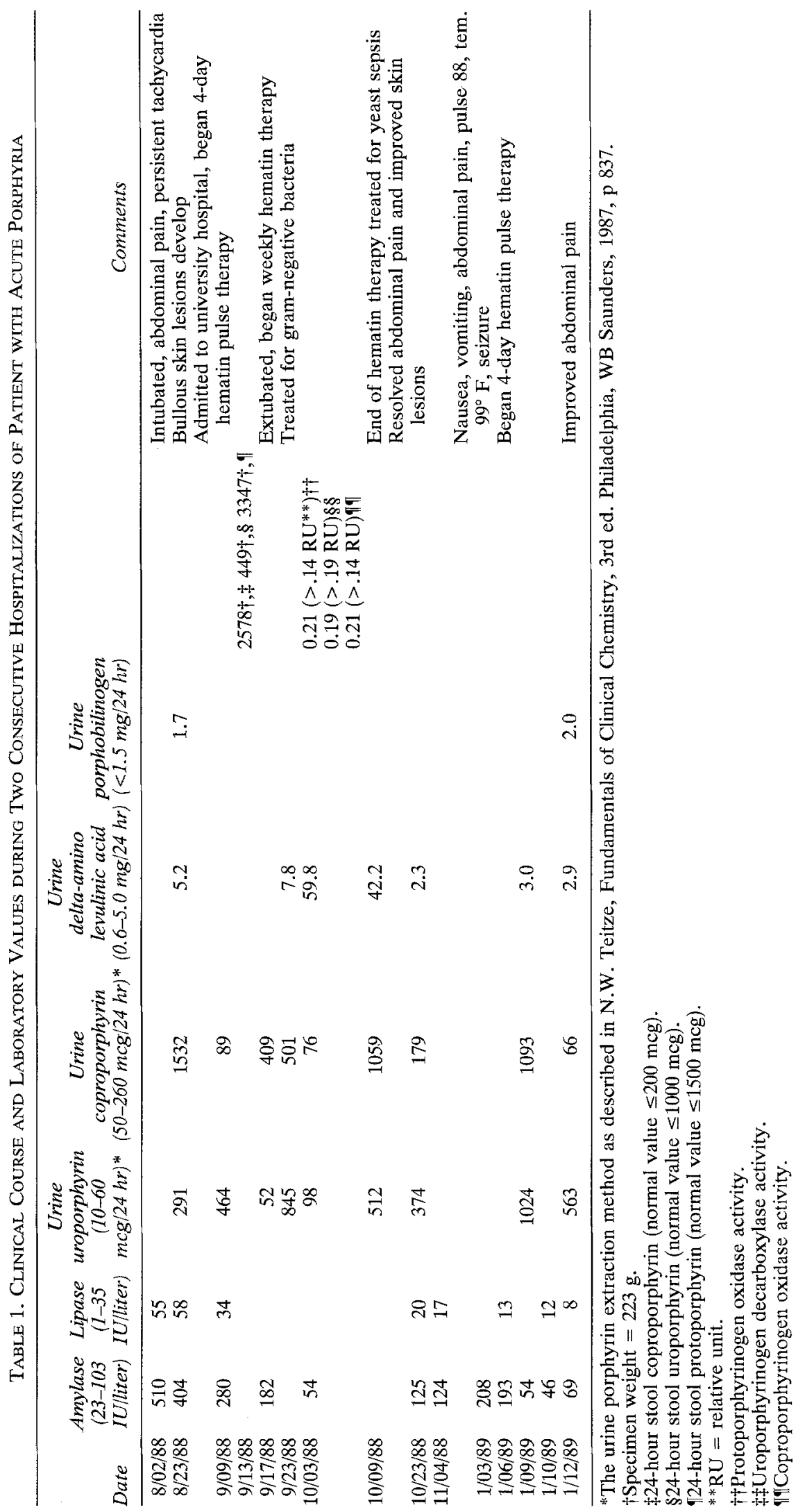


general downward trend with therapy and resolution of her clinical symptoms. The levels rose temporarily, however, associated with a gram-negative infection. She was discharged five months after her initial hospitalization.

Two months later after a period of decreased oral intake and mild dehydration due to probable viral gastroenteritis, she developed recurrent abdominal pain, nausea, vomiting, and had a seizure. She was readmitted for hematin pulse therapy and intravenous high carbohydrate feedings. Serum amylase values, again elevated early in her hospital course, were seen to resolve.

\section{DISCUSSION}

The only previous report of hyperamylasemia and porphyria concerns the occurrence of macroamylasemia and acute intermittent porphyria (1). The persistently elevated lipase values and elevated amylase-creatinine clearance ratio make macroamylasemia a highly unlikely explanation for the hyperamylasemia seen in this case $(2,3)$. With treatment of the porphyria, the patient's clinical symptoms, urinary quantitative porphyrins, and serum amylase decreased on two separate occasions. This certainly suggests an association between the porphyria and hyperamylasemia. However, the etiology of this elevated amylase remains unclear. The clinical course, radiographic evaluation, and laparotomy findings make acute pancreatitis unlikely. Subclinical mesenteric ischemia cannot be excluded, although the elevated amylase creatinine clearance ratio does not support this etiology (3).

Due to the timing of porphyrin measurements and the normal protoporphyrinogen oxidase activity, we cannot be certain which type of porphyria this patient had. However, the porphyrin measurements and clinical course clearly establish a diagnosis of porphyria and suggests the diagnosis of variegate porphyria.

The diagnosis and treatment of this case of porphyria associated with unexplained elevations of serum amylase and lipase were delayed because physicians were misled by the clinical and laboratory presentation suggesting acute pancreatitis. It is for this reason we wish to present this case showing an association of hyperamylasemia and hyperlipasemia with acute porphyria. Moreover, we wonder if others have seen such a relationship.

\section{SUMMARY}

An elevation of serum amylase and lipase has not been reported previously to occur with porphyria. In this report, we describe a patient who presented with the clinical and laboratory picture of pancreatitis: elevated amylase, lipase, amylase-creatinine clearance ratio, and with abdominal pain. Only after extensive evaluation, was the patient found to have porphyria. On two separate occasions, with hematin therapy, her serum amylase decreased, as did her clinical symptoms of porphyria and her urinary quantitative porphyrins. This suggests an association between elevation of the serum amylase and lipase with acute porphyria. Moreover, this association can lead to delay in establishing the diagnosis of acute porphyria.

\section{ACKNOWLEDGMENTS}

The authors would like to acknowledge Mrs. Jolene Amo, CMT, for her help in preparing this manuscript.

\section{REFERENCES}

1. Hedger RW, Hardison WGM: Transient macroamylasemia during an exacerbation of acute intermittent porphyria. Gastroenterology 60:903-908, 1971

2. Levitt MD: Clinical use of amylase clearance and isoamylase measurements. Mayo Clin Proc 54:428-431, 1979

3. Warshaw AL, Fuller AE: Specificity of increased renal clearance of amylase in diagnosis of acute pancreatitis. $\mathbf{N}$ Engl $\mathbf{J}$ Med 292:325-328, 1975 